\title{
Polynomial Evaluation on Superscalar Architecture, Applied to the Elementary Function $e^{x}$
}

\author{
TIMOTHÉE EWART, FRANCESCO CREMONESI, FELIX SCHÜRMANN, and \\ FABIEN DELALONDRE, Blue Brain Project, École Polytechnique Fédérale de Lausanne, Switzerland
}

\begin{abstract}
The evaluation of small degree polynomials is critical for the computation of elementary functions. It has been extensively studied and is well documented. In this article, we evaluate existing methods for polynomial evaluation on superscalar architecture. In addition, we have completed this work with a factorization method, which is surprisingly neglected in the literature. This work focuses on out-of-order Intel processors, amongst others, of which computational units are available. Moreover, we applied our work on the elementary function $e^{x}$ that requires, in the current implementation, an evaluation of a polynomial of degree 10 for a satisfying precision and performance. Our results show that the factorization scheme is the fastest in benchmarks, and that latency and throughput are intrinsically dependent on each other on superscalar architecture.
\end{abstract}

\section{CCS Concepts: • Mathematics of computing $\rightarrow$ Mathematical software performance;}

Additional Key Words and Phrases: Polynomial evaluation, compute units, superscalar architecture, elementary function

\section{ACM Reference format:}

Timothée Ewart, Francesco Cremonesi, Felix Schürmann, and Fabien Delalondre. 2020. Polynomial Evaluation on Superscalar Architecture, Applied to the Elementary Function $e^{x}$. ACM Trans. Math. Softw. 46, 3, Article 28 (September 2020), 22 pages.

https://doi.org/10.1145/3408893

The performance evaluation of small degree polynomials is a factor for the good execution and the performance of elementary functions. The theoretical research in polynomial evaluation is very well summarized in Knuth [1997] and Muller [1997]. In addition, the performance evaluation of these researches on the processors is always active from Overill and Wilson [1994] on the vectorial processor to Reynolds [2010], Dukhan and Vuduc [2013], and Shibata [2010].

On these examples, the study of Reynolds [2010] evaluates polynomial but does not put enough attention on pure performance (results should be reported in [cycles]), especially for small degrees. Dukhan and Vuduc [2013] only maximize the throughput of elementary functions, using a unique method of evaluation (Horner method) and does not provide any information on boundary limits

This study was supported by funding: (1) to the Blue Brain Project, a research center of the École Polytechnique Fédérale de Lausanne, from the Swiss government's ETH Board of the Swiss Federal Institutes of Technology and (2) through the Supercomputing and Modeling for the Human Brain (SMHB) project supported by the German Helmholtz Association. Authors' addresses: T. Ewart, F. Cremonesi, F. Schürmann (corresponding author), and F. Delalondre, Blue Brain Project, École Polytechnique Fédérale de Lausanne, Campus Biotech, Chemin des Mines 9., Genève, 1202, Switzerland; emails: \{timothee.ewart, francesco.cremonesi0\}@gmail.com, \{felix.schuermann, fabien.delalondre\}@epfl.ch.

Permission to make digital or hard copies of part or all of this work for personal or classroom use is granted without fee provided that copies are not made or distributed for profit or commercial advantage and that copies bear this notice and the full citation on the first page. Copyrights for third-party components of this work must be honored. For all other uses, contact the owner/author(s).

(c) 2020 Copyright held by the owner/author(s).

0098-3500/2020/09-ART28

https://doi.org/10.1145/3408893 
nor on the latency. Shibata [2010] focuses only on the throughput and also does not take into account boundary limits. HiPEAC [2015] provided an excellent work, but boundary conditions are again neglected. However, all these authors except Reynolds [2010] limit their polynomial evaluation to the classical Horner method. To summarize an excellent review of method of polynomial and elementary functions implementation can be found in Muller [1997].

In this article, we consider that the maximum performance can only be reached if the algorithm is specifically tailored for the hardware that it targets. Nowadays, in high-performance computing (HPC) the superscalar architecture and out-of-order execution are the de facto standard, so the algorithm must be parallel to maximize the pipeline utilization. Consequently, at the time scale (tens of cycles) of the low order polynomial evaluation, algorithms with higher parallelism may outperform others, in spite of having a larger operation count. Of course, this difference might not exceed a few cycles, but for such a fundamental operation that may still have a large impact.

The work of Abbas and Gustafsson [2011] offers an interesting perspective on the performance of several polynomial evaluation schemes by performing a theoretical analysis of the number of operations, critical path, latency, and pipelining. While this analysis provides very useful insight into the properties of different schemes, it lacks benchmarks on modern architectures, whose performance is notoriously complex and hard to predict. On state-of-the-art HPC architectures, the selection of the best algorithm for a given task must be tied to the hardware under consideration and can only be finalized through automatic benchmarking [Balaprakash et al. 2018]. Indeed, in the past decade a lot of effort has been put into automatic generation of code for polynomial evaluation, a technique that allows to directly benchmark the performance of a large number of possible evaluation schemes, as shown in Mouilleron and Revy [2011] and Fateman [2002]. In particular, the CPGE tool ${ }^{1}$ presented in Mouilleron and Revy [2011], which combines heuristics and benchmarking to select the best-performing method for a given architecture, is very relevant for our work. However, we focus mainly on HPC architectures based on Intel processors, while benchmark results in Mouilleron and Revy [2011] were presented for dedicated hardware.

This work focuses on the evaluation of degree 10 polynomials, which represent a good compromise between precision and performance, in double precision only, on superscalar out-of-order processors, and their application to the elementary function $e^{x}$. In Section 1, we review the stateof-the-art of polynomial evaluation and in Section 2 the computation of the elementary function $e^{x}$. While a review on the methods of evaluation of elementary functions is outside the scope of this article, we refer the reader to Muller [2006] for a comprehensive description of the problem, and to Saint-Genies [2018] for a recent review with a focus on performance on SIMD-enabled, superscalar architectures. Then, more technically, we describe the superscalar architecture and core units of the Intel's x86 processor (Sandy Bridge and Broadwell) and the different methods we will use to code and measure the veracity of the results. We present results for the polynomial evaluation and exponential for scalar and vectorial versions and compare it to the Intel vendor's exponential library.

\section{POLYNOMIAL EVALUATION, INTRODUCTION}

In evaluating the polynomial of degree $n$,

$$
P(x)=a_{0}+a_{1} x+\cdots+a_{n} x^{n},\left(a_{i} \in \mathbb{R}\right),
$$

a large number of methods can be applied. In this study, we selected the most significant, extracted from the work of Knuth [1997] who collected and summarized all the state-of-the-art. The following methods were selected: the Brute Force method, the Horner method from order 1 to $n-1$, and

\footnotetext{
$\overline{{ }^{1} \text { see http://cgpe.gforge.inria.fr/. }}$
} 
Table 1. Number of Operations for Different

Algorithms Used for the Evaluation of a Polynomial of Degree $n$

\begin{tabular}{lcc}
\hline & addition & multiplication \\
Brute force & $n$ & $2 n-1$ \\
Horner $k$ th-order & $n$ & $n+k-1+\theta(k)$ \\
Estrin & $n$ & $n+\left\lfloor\log _{2}(n)\right\rfloor$ \\
Factorization & $n$ & $\left(\sum_{i=1}^{n} m_{i}\right)+n-1$ \\
\hline
\end{tabular}

The number of multiplications of the factorization depends of the number of the method selected associated to its complexity for the "sub" polynomials formed by the quadratics.

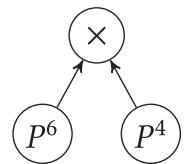

(a) $P^{10}=P^{6} P^{4}$

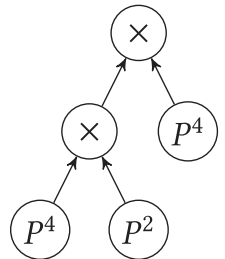

(b) $P^{10}=P^{4} P^{2} P^{4}$

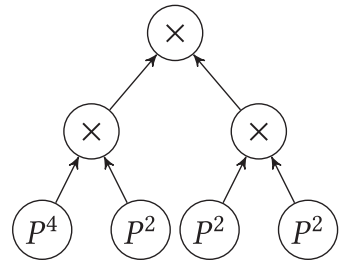

(c) $P^{10}=P^{4} P^{2} P^{2} P^{2}$

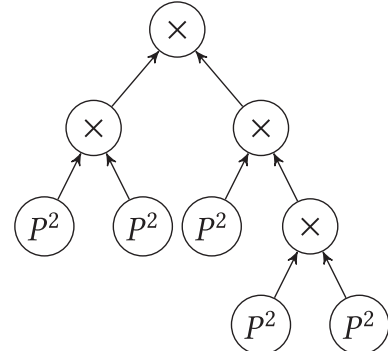

(d) $P^{10}=P^{2} P^{2} P^{2} P^{2} P^{2}$

Fig. 1. The five quadratics of the polynomials of degree 10, i.e., $P^{10}$ (Equation (8)) can be recombined in polynomial of higher degree, e.g., $P^{10}=P^{6} P^{4}$ or $P^{10}=P^{4} P^{2} P^{2} P^{2}$ and so on.

the Estrin method. Moreover, factorization techniques were applied to all the methods previously enumerated. Several other methods for evaluating polynomials have been demonstrated in the literature, mainly exploiting use-case specific optimizations to achieve the near-optimal number of operations. Most notably, preconditioning methods exploit the a priori knowledge of the polynomial to find a transformation of the coefficients that allows to improve performance. Examples of these methods are Knuth's adaptation of coefficients [Knuth 1962], Winograd's method [Rabin and Winograd 1972], and others [Ercegovac 1977; Fateman 2002; Lipton and Stockmeyer 1978]. Such methods are in a sense close to the factorization strategy of decomposing the original polynomial into sub-polynomials. These preconditioned methods are not incompatible with our work, and both methods are in a sense overlapping. However, the goal of this article is showing that factorization is a viable approach for polynomial evaluation on superscalar architectures.

The classical approach to compare the performance of numerical schemes is to analyze the number of required operations. In Table 1 , we summarize the total number of operations $(\times,+)$ for all methods under consideration in this study. In this table the factorization is a combination of the $n$ different methods. Each of the $n$ factors in the factorization can be evaluated with one of these methods; the total number of multiplications will be $n-1$ plus the sum of the number of multiplications required for each factor (see Figure 1). As modern processors become more and more complex, exposing instruction-level parallelism and super-scalar operations, the relationship between number of operations and performance becomes less and less clear. To improve performance on modern multicore, multi-scalar architectures, the main challenge is to maximize parallelism and minimize the pipelining data hazards to avoid bubbles that have the effect of deteriorating performance. 
In the rest of this section, we present the main features and complexity (measured in number of operations) for a selected subset of the most common polynomial evaluation schemes. Finally, in Section 1.5, we present our novel approach based on factorization.

\subsection{Brute Force}

The brute force method simply consists of evaluating the polynomial (1), term after term. To compute every monomial $x^{n}$, the Exponentiating by squaring method [Knuth 1997] is implemented. The method is based on the observation that, for a positive integer $n$, we have

$$
x^{n}= \begin{cases}x\left(x^{2}\right)^{\frac{n-1}{2}} & \text { if } n \text { is odd } \\ \left(x^{2}\right)^{\frac{n}{2}} & \text { if } n \text { is even. }\end{cases}
$$

To compute an arbitrary polynomial of degree $n$, all the monomials must be explicitly evaluated; we can expect the compiler to perform the necessary optimizations. Therefore, monomials already determinate will be not recomputed, reducing the multiplications of all the individual monomials to the minimum required of $n-1$. Once all the individual monomials have been obtained, this method requires an additional $n$ coefficient multiplications, for a total of $2 n-1$ multiplications, to evaluate the full polynomial.

\subsection{Horner Method: Classical - 1st-order}

The first-order Horner method is among the most widely studied schemes in the literature, arguably for its simplicity and efficiency. Given an input value $x_{0}$, it recursively evaluates the polynomial (1) as

$$
P\left(x_{0}\right)=a_{0}+x_{0}\left(a_{1}+x_{0}\left(a_{2}+x_{0}\left(\ldots a_{n}\right)\right)\right) .
$$

"Horner's method" needs $n$ multiplications and $n$ additions, or $n$ Fused Multiply-Add (FMA). This method requires a relatively low number of addition and multiply operations, but is inefficient from the point of view of the processor, because it introduces pipelining data hazards due to the dependency chain in the recursive process.

\subsection{Horner Method: $k$ th-order}

"Horner's method" can be generalized to an arbitrary order by building new polynomials over $x^{k}$ instead of $x$ [Dorn 1962]. The main advantage is to introduce parallelism that removes data hazards, especially for high orders of $k$, e.g., $k=2$ corresponds to the "Even-Odd" split [Newbery 1975]. Given a degree of parallelism $k$, Horner's $k$ th-order method reads:

$$
\begin{aligned}
P\left(x_{0}\right) & =\sum_{m=0}^{k-1} Q_{m} x_{0}^{m}, \\
Q_{m} & =\sum_{j=0}^{\lceil n / k\rceil-1} a_{k j+m} x_{0}^{k j} .
\end{aligned}
$$

Developing Equation (4) to the "Even-Odd" pattern $(k=2)$ leads to:

$$
\begin{aligned}
P\left(x_{0}\right) & =Q_{0}+Q_{1} x_{0} \\
Q_{0} & =a_{0}+x_{0}^{2}\left(a_{2}+x_{0}^{2}\left(a_{4}+x_{0}^{2}\left(a_{6}+x_{0}^{2}\left(\cdots+x_{0}^{2} a_{k j}\right)\right)\right)\right. \\
Q_{1} & =a_{1}+x_{0}^{2}\left(a_{3}+x_{0}^{2}\left(a_{5}+x_{0}^{2}\left(a_{7}+x_{0}^{2}\left(\cdots+x_{0}^{2} a_{k j+1}\right)\right)\right) .\right.
\end{aligned}
$$

This method requires $n$ additions and $n+k-1+\theta(k)$ multiplications, where $\theta(k)$ contains the sum of all the multiplications needed to compute the monomials $x^{m}, m=2, \ldots k$. The classical 
Horner method corresponds to $k=1$. In this article, the Horner method will be evaluated until order 9.

\subsection{Estrin Method}

An alternative to the generalization of Horner's rule is the Estrin method [Estrin 1960], based on the following splitting for an arbitrary polynomial $P^{n}$ of degree $n$ :

$$
P^{n}=x^{\lceil n / 2\rceil} Q^{\lfloor n / 2\rfloor}+R^{\lfloor n / 2\rfloor},
$$

where $Q^{\lfloor n / 2\rfloor}, R^{\lfloor n / 2\rfloor}$ are polynomials whose coefficients are obtained directly from Equation (1). It is possible to further recursively apply the splitting in Equation (6) to the polynomials $Q, R$ in a divide-and-conquer approach. The expression thus obtained corresponds to the application of Estrin's scheme. For example, developing Equation (6) leads to:

$$
\begin{aligned}
P\left(x_{0}\right)= & a_{0}+a_{1} x_{0} \\
& +x_{0}^{2}\left(a_{2}+a_{3} x_{0}\right) \\
& +x_{0}^{4}\left(a_{4}+a_{5} x_{0}+x_{0}^{2}\left(a_{6}+a_{7} x_{0}\right)\right) \\
& +x_{0}^{8}\left(a_{8}+a_{9} x_{0}+x_{0}^{2}\left(a_{10}+a_{11} x_{0}\right)\right. \\
& \left.\left.+x_{0}^{4}\left(\left(a_{12}+a_{13} x_{0}+x_{0}^{2}\left(a_{14}+a_{15} x_{0}\right)\right)\right)\right)\right) \\
& \cdots
\end{aligned}
$$

This method requires $n$ additions and $n+\left\lfloor\log _{2}(n)\right\rfloor$ multiplications but has a remarkable pattern design for the following reason: At the last recursion step, every subpolynomial $Q^{1}, R^{1}, \ldots$ can be associated to an independent FMA, and by virtue of this independence, we expect a good efficiency on superscalar machines (i.e., no bubbles in the pipeline).

\subsection{Factorization}

Finally, we consider the factorization method. This method is based on the fundamental algebraic property of polynomials with real coefficients [Klein 1932], which states that any polynomial $P(x)$ of degree $2 n$ can be decomposed into a product of $n$ quadratic factors with real coefficients (factorization):

$$
P(x)=\prod_{j=1}^{n}\left(x^{2}+\alpha_{j} x+\beta_{j}\right) .
$$

Instead of evaluating all the simple factors appearing in Equation (8) one-by-one, we consider all their possible combinations into polynomials of higher degree, to test whether a particular sequence of higher degree polynomials yields better results. Figure 1 illustrates possibilities of the combinations to obtain a polynomial of degree 10. Each higher degree polynomial can be evaluated by one of the previously described methods. Moreover, for the evaluation of each polynomial factor in Equation (8), we consider all the techniques described above. Section 2.1 demonstrates that the total number of combinations is equal to 231. In this article, no preconditioning is performed. The coefficients $\alpha_{j}$ and $\beta_{j}$ have been determinated using third-party software (Matlab). The factorization technique can introduce a large degree of parallelism and may outperform others for superscalar architectures. The factorization method can be easily extended to polynomials of odd degree; in such case, a preliminary step consists of factorizing by $x$ and applying our method on the resulting even degree polynomial, i.e., $P^{2 n+1}(x)=x P^{2 n}(x)+a_{0}$. 


\section{ELEMENTARY FUNCTION: $\operatorname{EXP}(X)$}

The evaluation of elementary transcendental functions is a widely studied topic that has many applications [Muller 2006; Saint-Genies 2018]. Function implementation methods based on a table lookup have been proposed for the exponential function [Tang 1989], trigonometric functions [de Lassus Saint-Genies et al. 2017] and elementary functions in general [Tang 1991]. However, these methods are typically not SIMD-friendly ${ }^{2}$ and may require additional work to prevent the propagation of numerical error, so they were not considered in this article where we focus on methods that can be readily and efficiently utilized on superscalar architectures. Since most approximations of functions are valid in a small interval only, a common first step is to perform range reduction, which consists in reducing the range of input values by algebraic manipulation. Since this work focuses on the exponential function, we provide an example below:

$$
\begin{gathered}
\forall x \in \mathbb{R}, \exists y \in[0, \ln 2[\text { with } k \in \mathbb{N} \text { such that: } \\
\qquad e^{x}=2^{k} e^{y} .
\end{gathered}
$$

The restriction on the range $\left[0, \ln 2\left[\right.\right.$ simplifies the evaluation of $e^{y}$. To evaluate $e^{y}$, a truncated Taylor series represents a straightforward approach; however, a polynomial approximation is more valuable, as described in the next paragraphs.

Truncated series. The Taylor series of $e^{y}$ can be expressed as the sum of an approximation and a truncation error:

$$
e^{y}=\underbrace{\overbrace{1+y+\frac{y^{2}}{2 !}+\cdots+\frac{y^{n}}{n !}}^{\text {Approximation }}+\overbrace{\frac{y^{n+1}}{n+1 !}+\cdots}^{\text {Truncation Error }}}_{\text {Exact mathematical formulation }} .
$$

The "Truncation Error" is the remainder $R_{n}$ from Taylor's Theorem [Gerald and Wheatley 2004]. It can be expressed and bounded under the Lagrange form: $R_{n} \leq\left|\frac{e^{y}}{(n+1) !} y^{n+1}\right|$. When $y=\ln 2$ and $n=10$, the remainder term has a magnitude $R_{10} \leq\left|\frac{e^{l n 2}}{11 !} \ln (2)^{11}\right| \sim 8.89 \times 10^{-10}$, which is a large error compared to floating point accuracy. According to this formula, to guarantee an error lower than the double precision of the machine, $n=16$ is needed. However, it is beyond our objective of $n=10$. A more accurate alternative to the Taylor series is, e.g., the Pade approximant [Baker and Graves-Morris 1996], but it requires a division that is very time-consuming.

Polynomial approximation. An alternative is to approximate $e^{x}$ with a polynomial using a minimax method. The Remez algorithm is an excellent solution. This method certifies that $f(x)\left(e^{x}\right.$ in our case) can be approximated by a unique minimax solution $R(x)$ (a polynomial of degree $n=10$ ) minimizing an error function, e.g., $E(x)=f(x)-R(x)$ in the concerning interval.

The Remez method is powerful; a detailed and rigorous analysis can be found in Fraser [1965]. In this work, the Remez method was performed with a customized extended form of the floating point (150 bits digit precision [Software 2012]) to avoid penalizing rounding effect during the computation of the Remez method. The final 150 bit coefficients are rounded to 64 bits double precision. However, it gives a satisfactory trade-off between accuracy and performance (demonstrated in Section 4.3). Additionally, to increase accuracy, future investigation could be performed using the Sollya library [Chevillard et al. 2010]. This would permit avoiding the rounding step and potentially improve the final accuracy.

\footnotetext{
${ }^{2}$ Since the writing of this article a new SIMD-friendly implementation of elementary functions has been released [Lauter 2016].
} 


\subsection{Combinatory}

The factorization method and the different polynomial evaluation techniques described above give rise to a large number of possible combinations of polynomial factors. We now present a method for computing exactly how many different combinations we need to consider.

In this study, we focus on the evaluation of polynomials of degree 10, where the coefficients are known in advance. We concentrate specifically on these, because they are very useful in the approximation of the exponential function $e^{x}$ with $x \in[0, \ln 2[$. The polynomials we consider are strictly positive in the range $[0, \ln 2[$, and therefore do not have any real roots, but five pairs of complex conjugate roots. From the five pairs, five quadratic polynomials can be built following the factorization process described above. The product of these five quadratic factors is the factorization scheme. We note alternative schemes exist.

Before doing the combinatory analysis, let us introduce the following notation: Any polynomial $P$ is characterized by two parameters, the degree and the method of evaluation. The degree is represented by the superscript $n$, whereas the subscript is the method of evaluation. The different methods of evaluation are the brute force method $(b)$, the Horner method of order $k\left(h^{k}\right)$, and the Estrin method $(e)$. For example, $P_{e}^{6}$ means a polynomial of degree 6 evaluated with the Estrin method, whereas $P_{h^{2}}^{8}$ means a polynomial of degree 8 evaluated with the Horner method with order 2 .

To find the total number of combinations of polynomial factors, we start by considering all possible partitions of the number 10 as a sum of even numbers. We only consider even numbers, because, as stated above, all the polynomial factors we consider are of degree two, arising from the product of two factors associated to complex conjugate roots. Partitioning the number ten in even summands is equivalent to partitioning the number five into arbitrary summands, then multiplying by two. There are seven partitions of the number five, which are, using conventional notation, $(5),(4,1),(3,2),\left(3,1^{2}\right),\left(2^{2}, 1\right),\left(2,1^{3}\right),\left(1^{5}\right)$. Consequently the partitions of ten into even summands are

$$
(10),(8,2),(6,4),\left(6,2^{2}\right),\left(4^{2}, 2\right),\left(4,2^{3}\right) \text {, and }\left(2^{5}\right) .
$$

To evaluate the number of combinations, three considerations are made:

(1) each polynomial $P^{m}$ has $m+1$ methods of evaluation: $P_{b}^{m}, P_{e}^{m}$, and $P_{h^{k}}^{m}$ where $k \in[1, m-$ 1] because $P_{h^{1}}^{m}=P_{h^{n}}^{m}$;

(2) for each polynomial product $P^{l} P^{m}$ with $l \neq m$, there are $(l+1) \times(m+1)$ possible combinations (we do not consider the alternative $P^{m} P^{l}$ because multiplication is commutative);

(3) for each product of $k$ polynomials having the same degree $m$ there are $\left(\begin{array}{c}m+k \\ k\end{array}\right)$ and not $\left(\begin{array}{c}m+1 \\ k\end{array}\right)$ possible combinations, because repetitions are not counted.

Applying this scheme to Equation (11) determines the total number of combinations $S$ :

$$
\begin{aligned}
S & =\operatorname{card}\left((10)+(8,2)+(6,4)+\left(6,2^{2}\right)+\left(4^{2}, 2\right)+\left(4,2^{3}\right)+\left(2^{5}\right)\right) \\
& =11+9 \times 3+7 \times 5+7 \times\left(\begin{array}{l}
4 \\
2
\end{array}\right)+3 \times\left(\begin{array}{l}
6 \\
2
\end{array}\right)+5 \times\left(\begin{array}{l}
5 \\
3
\end{array}\right)+\left(\begin{array}{l}
7 \\
5
\end{array}\right) \\
& =231 .
\end{aligned}
$$

The total number of combinations to evaluate $P^{10}$ is therefore 231 .

\subsection{From $\mathrm{x}$ to $\mathrm{k}$ and $\mathrm{y}$}

The polynomial evaluation issue solved, let us go back to the $e^{x}$ computation. The Equation (9) can be solved although it has two unknowns.

$\forall x \in \mathbb{R}, \exists y \in[0, \ln 2[, k \in \mathbb{N}$ such that:

$$
x=y+k \ln 2 .
$$


To determine $k$, we apply the floor function to both sides of Equation (13)

$$
\begin{aligned}
\left\lfloor\frac{x}{\ln 2}\right\rfloor & =\underbrace{\left\lfloor\frac{y}{\ln 2}\right\rfloor}_{=0}+\underbrace{\lfloor k\rfloor}_{=k} \\
& =k .
\end{aligned}
$$

Now that $k$ has been determined, we can compute $y$ by solving Equation (13).

The previous equations can be integrated in the next algorithm. However, the algorithm may introduce rounding error [Goldberg 1991] due to the multiplication between the large gap between $k$ and $\ln (2)$.

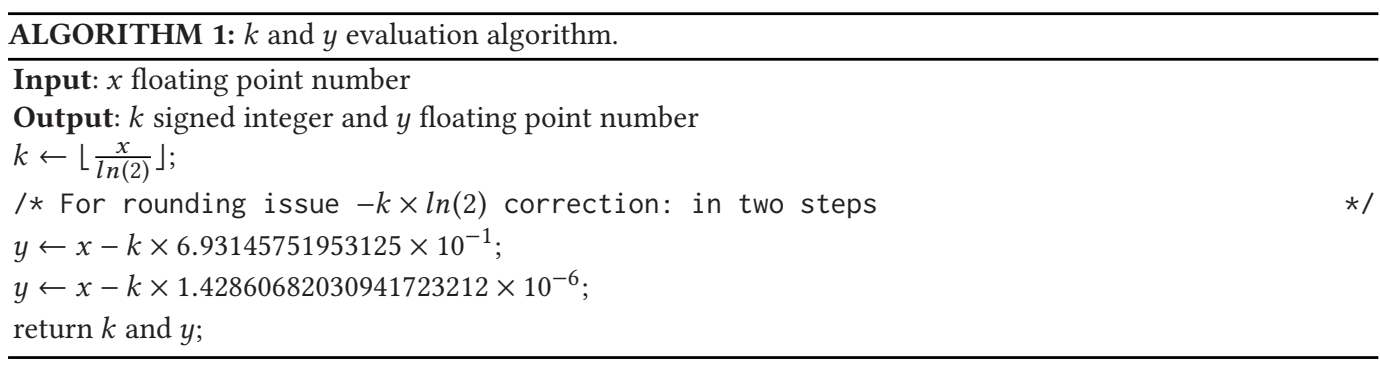

The double-double arithmetic [Joldes et al. 2017; Lauter 2005] method is applied, where $\ln (2)$ is split into two floating-point numbers $c_{h}$ and $c_{l}$ of a smaller significant size. Then the expression $k \ln (2)$ can be approximated by $k c_{h}+k c_{l}$. The coefficients $c_{h}$ and $c_{l}$ have been extracted from the cephesibrary [Moshier 2000]: $c_{h}=6.93145751953125 \times 10^{-1}$ and $c_{l}=1.42860682030941723212 \times$ $10^{-6}$.

\section{$2.32^{k}$ Fast Evaluation}

Computing $2^{k}$ may be done with a left bit shift. However, it is not suitable because of the limited domain with 64-bit integer. An alternative solution utilizes the floating point representation. Real numbers can be represented approximately under the following form:

$$
x=-1^{s} \times(1+F) \times 2^{e+b i a s} .
$$

For IEEE-754 double precision number: $s$ sign bit, $e+$ bias is the exponent (11 bits). This has a bias (in the engineering sense of the word) equal to 1,023 , and $e$ belongs $[-1,022,1,023] . F$ is the mantissa (52 bits) $\sum_{n=1}^{p-1} b i t_{n} \times 2^{-n}$. The method consists of taking the desired $k$, adding the bias and moving it into the exponent part of the floating point representation, and letting the machine interpret it like a double. The method is efficient, fast, and does not introduce any errors, overflows, if obviously the boundary conditions of $x$ are respected (2.4). This method can be described by the following algorithm:

ALGORITHM 2: $2^{k}$ evaluation algorithm. Note the operation interpret_as_double interprets $k$ as a double; it is not a cast operation.

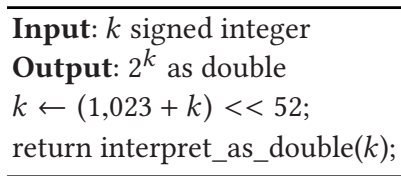


Table 2. Boundary of the Exponential for Double Precision

\begin{tabular}{ccccccc}
\hline$x$ & $-\infty$ & $+\infty$ & 0 & $\mathrm{NaN}$ & $>709.09$ & $<-709.09$ \\
\hline$e^{x}$ & 0 & $+\infty$ & 1 & $\mathrm{NaN}$ & $+\infty$ & 0 \\
\hline
\end{tabular}

\subsection{Boundary Limits}

The last step consists of fixing the boundary limits. This step is mandatory, and it introduces additional workflows, although it has been sometimes neglected in previous studies on the exponential function [Dukhan and Vuduc 2013; HiPEAC 2015; Shibata 2010]. For double precision, the boundary limits to respect are summarized in the Table 2.

Branching is impossible on SIMD architectures; nevertheless, we describe an alternative using bitwise manipulation, as described in Algorithm 3.

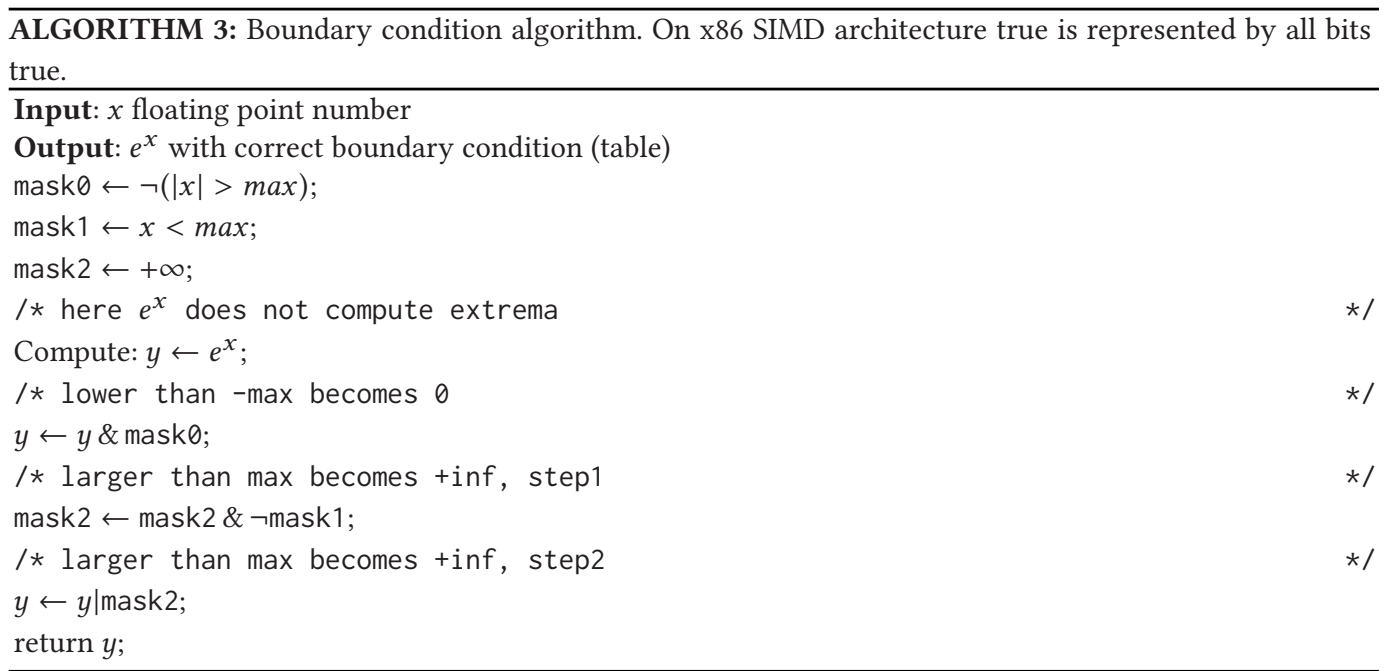

This algorithm has been applied to the scalar and vectorial versions. An alternative could be a simple branching on the absolute value of $x$ : if $(|x|<707.09)$ do $\exp (x)$ else if $(\ldots)$. In the next sections, for each benchmark, $1 \mathrm{~K}$ inputs were generated using an uniform distribution over the range [-707.09,707.09], consequently the branching is reduced to a minimum. In such case, the cost of the branching (inequality, absolute value and "jump") is more or less 2 cycles. Since branch predictors are efficient in such cases, the scalar version with a standard branching implementation may outperform the scalar version with mask implementation. However, we decided to keep the latter for a more consistent comparison. The overhead of masking is visible in the boundary column of the Table 7. To conclude, Intel released for its latest product native conditional SIMD instructions that should improve the performance, and a full investigation of branching and the performance of the predictor is a tough task, as it is demonstrated in the last survey of the technology [Mittal 2018], or the microprocessor branching unit description of Fog [2016].

\section{SUPERSCALAR PROCESSORS \& METHODS}

\subsection{Latency, Throughput Definitions}

This section provides the definitions of the main concepts described in the next sections.

Dependency chain: a dependency chain is a series of operations. Each operation needs the computation of the previous one to be computed. 
Table 3. Latency/Throughput add/mul/fma on the Sandy Bridge and Broadwell Architecture

\begin{tabular}{lcccc}
\hline & \multicolumn{2}{c}{ Sandy Bridge } & \multicolumn{2}{c}{ Broadwell } \\
& Thr. & Lat. & Thr. & Lat. \\
\hline add & 1 & 3 & 0.8 & 3 \\
mul & 1 & 5 & 0.5 & 5 \\
FMA & - & - & 0.5 & 5 \\
\hline
\end{tabular}

These values are the same for scalar and vectorial mnemonics.

Pipeline and pipelining: a pipeline is a technical implementation (in hardware) in which multiple instructions are overlapped in execution.

Latency: The latency of an instruction is the delay that the instruction generates in a dependency chain.

Throughput: The throughput is the maximum number of instructions of the same kind that can be executed per clock cycle when the operands of each instruction are independent of the preceding instructions.

In what follows, we report the inverse throughput that is instead measured in [cycle] for consistency reasons: In this way, both latency and inverse throughput values have the same unit of measurement, and for both smaller values are best. The latency and the throughput of the main instructions for all platforms are reported in Table 3.

\subsection{Architecture Details}

Several definitions of superscalar processor exist, but two seem more interesting: Dispatch multiple instructions every cycle [Center et al. 1987] or Execute two or more scalar operations in parallel [Lam 1990]. Whatever the definitions, they are two main ideas: execute instructions concurrently and independently in separate execution pipelines. Moreover, the throughput of the execution into the concurrent pipelines may be much improved by allowing out-of-order execution. Nevertheless, if the dispatcher is ineffective at keeping all of these units fed with instructions, the performance of the system will not be better than that of a simpler, cheaper in-order design.

Modern processors have more than one pipeline. Complementary to the execution pipelines, the execution of a single instruction is also a pipeline with several steps [Boersma et al. 2011]. It allows restarting of the same operation (if no dependency chain) without waiting for the completion of the previous one.

Performing a full review of processors is outside the scope of this article. We focus on the basic execution of the processors, but still consider enough details to explain our results. Whatever the architecture of the chip, the execution of the binary code follows the same logic. Fetch/decode units read a stream of instructions from the L1 instructions cache memory and decode them into a series of micro operations that are executed by the processor's parallel executions units. The next paragraph takes an excerpt of the great work of Fog [2016] describing all x86 architectures.

The Sandy Bridge and Broadwell are out-of-order architectures supporting the new set of 256 bit vectorial instructions named AVX and AVX2 (Broadwell only). AVX2 provides FMA support and better integer and bitwise manipulation. The registers are generic, unified, and nested: It means modern, (i.e., 386 and beyond) x 86 processors have all the legacy registers nested into a bigger one, i.e., MMX registers (64 bits) are nested into SSE (128 bits) that are nested into AVX registers (256 bits). 
The Broadwell processor has eight pipelines, two Floating Point Units (FPUs), and four Arithmetic Logic Units (ALUs), while Sandy Bridge has six pipelines, two FPUs, and three ALUs. APU and ALU are polyvalent whatever the register, consequently, the floating-point operations have same latency and throughput per register (scalar or vectorial). The pipelines dedicated to floating point operations are numbers 0 and 1 on both architectures.

\subsection{Precision}

We compute the precision using the Unit of Least Precision (ULP) [Muller 2005] metric. The standard definition is: $u l p(x)$ is the gap between the two floating-point numbers closest to $x$, even if $x$ is one of them. The precision for the implementation of the exponential function will depend only on the error arising from the polynomial evaluation, because the evaluation of $2^{k}$ uses the exact hardware representation of the floating point numbers, and the boundary conditions only introduce bitwise manipulation. Here, the ULP has been computed using the boost library, specificially the boost: : math package.

\subsection{Measurement Tools}

Measuring the throughput/latency is challenging, especially for the latency. A direct measurement is not trivial due to the large number of variables that may impact the measurements. Therefore, the approach used within this study consists of performing indirect measurements by mapping the instructions associated to their mnemonics into a for loop whose unroll factor was parameterizable (between 1 and 5). To avoid introducing some operation throughput effect that would corrupt the measurement of latency, a dependency between instructions throughout the loop iterations has been artificially enforced. This method of measurement has been tested with success on the Power8 machine [Ewart et al. 2015]. Total control of the generated code and a good understanding of the $\mathrm{CPU}$ states is necessary, because the compiler may introduce unwanted effect like reorganizing inline assembly block, and so on.

Within this study, all benchmarks were compiled using GCC and repeated 100 times. The experimental data were fitted to a linear distribution $(y=a x+b)$ where $a$ corresponds to the slope of the curve that is equal to 100 times the actual latency of the instruction. Latency measurements were carried out for all basic instructions,$+ \times$, and FMA and compared to the vendor results to validate our method successfully. Latency of the previous instructions are reported in Table 3.

A full review of all x86 instructions can be found in Fog [2016]. To conclude, Intel previously released the IACA tool [Intel 2012]. This tool is nice for performing latency and throughput analysis and obtaining predictions on several Intel processor targets. The tool has been utilized to generate the Direct Acyclic Graph of a few polynomials evaluations on the Broadwell platform (Figure 2).

\subsection{Programming Model}

Programming polynomial evaluations and $e^{x}$ for high performance computing requires high standard code, because the code cannot be polluted by additional workload and useless instructions. A solution would be to write directly in assembly language [Goto and Geijn 2008]; this solution is conceivable although it is very time-consuming. We consider the alternative of using $\mathrm{C}++$ metaprogramming associated to recursive algorithms, inspired from the famous pattern of the metafactorial [Vandevoorde and Josuttis 2002]. It simplifies the generation of all polynomial evaluations and the code is generic. The algorithms are successively instantiated with the basic type double and a vector class from Ewart et al. [2014] or any of those from Pohl et al. [2016]. To control the quality of our ASM, at least on an x86 platform, we extract the DAG (Figure 2) using the IACA tool, for example for the evaluation of the first-order Horner method on a polynomial of degree 10. The match is perfect between the theory and the generated assembly. 


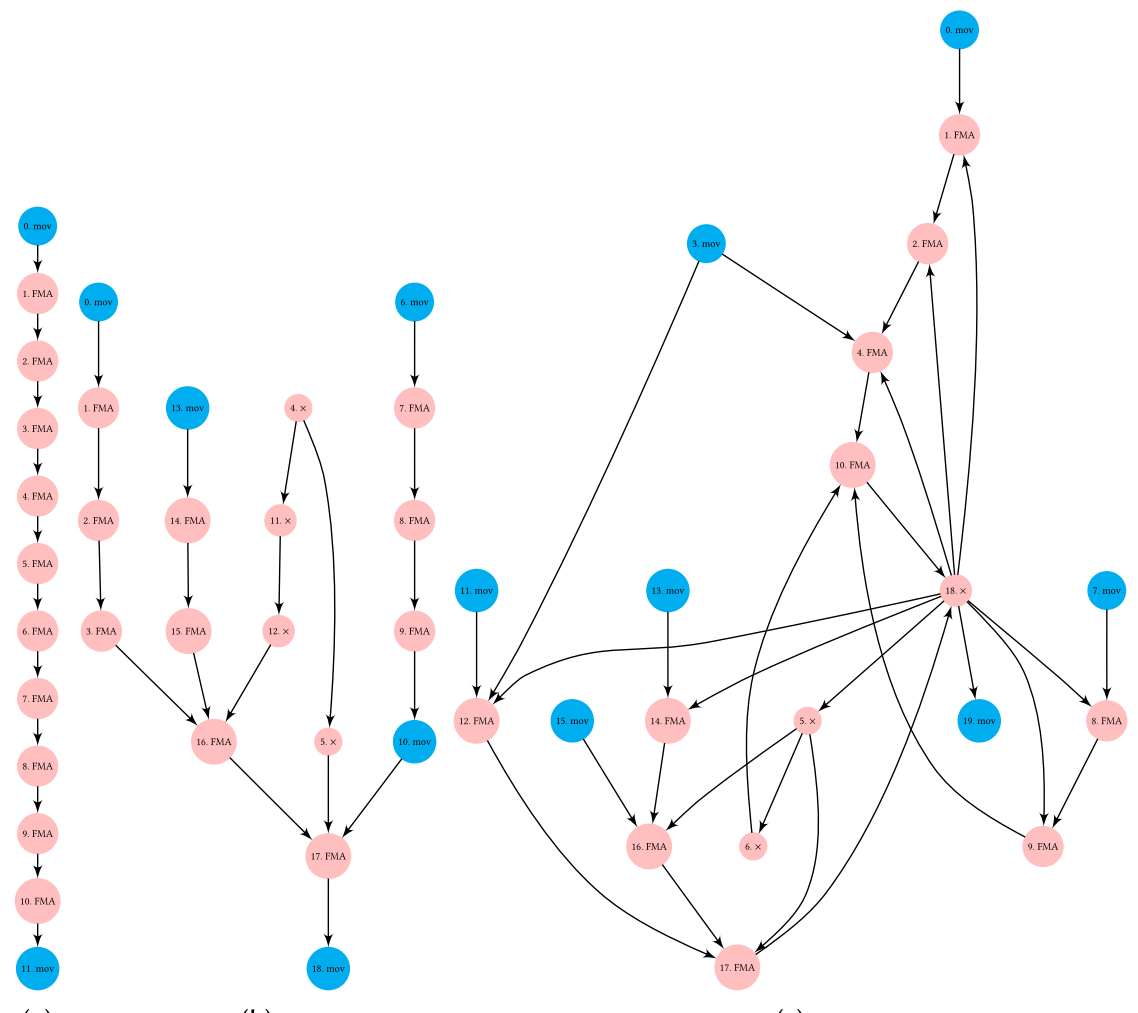

(a)

(b)

(c)

Fig. 2. Broadwell mnemonic DAG of the polynomial evaluation for three methods of evaluation (latency/ inverse throughput), (a) classical Horner (50.0/4.09 [cycle]), (b) Estrin (22.5/3.31 [cycle]), (c) Estrin $^{6} \times$ BruteForce $^{4}(24.00 / 3.85$ [cycle]).

Table 4. Description of the Processors, the Compilers, and the Compilation Options

\begin{tabular}{lcccc}
\hline $\begin{array}{l}\text { Architecture } \\
\text { Sandy Bridge }\end{array}$ & $\begin{array}{c}\text { Model } \\
\text { E5-2670 }\end{array}$ & $\begin{array}{c}\text { FMA } \\
\text { no }\end{array}$ & $\begin{array}{c}\text { Compiler } \\
\text { GCC 4.9 }\end{array}$ & $\begin{array}{c}\text { Options Compilation } \\
\text {-03 -march=native } \\
\text { Broadwell }\end{array}$ \\
E5-2630 & yes & GCC 4.9 & $\begin{array}{c}\text {-03 -march=native -fma } \\
\text {-fast-math -fabi-version=6 }\end{array}$ \\
\hline
\end{tabular}

\subsection{Processors and Compilers}

This work focuses on the last release of Intel Xeon processor (Broadwell) and an oldest generation (Sandy Bridge). The Intel architecture has been privileged, because it is well known and well documented. The compiler is GCC. All information is summarized in Table 4.

The option -fast-math reorders the algebraic associative expressions following the IEEE-754 standard for a faster evaluation. Although this method is agressive, it was mandatory to significantly improve the performance of the scalar version. Note that this optimization does not work for the vectorial version, since compilers do not reorganize SIMD code, i.e. every intrinsic is considered as it is placed by the programmer. The - fma is mandatory to catch FMA patterns. Finally, the option - fabi-version $=6$ is used for GCC vector Instructions through library signature. 
Table 5. Best Method (Factorization, Horner, or Estrin) for the Polynomial $P(x)$ and Exponential $e^{x}$ Evaluations on Sandy Bridge and Broadwell Platform

Following the Criteria (Throughput, Latency, ULP)

\begin{tabular}{lcccc|ccc}
\hline & & \multicolumn{3}{c|}{ Scalar } & \multicolumn{3}{c}{ Vector } \\
& & Th. & La. & ULP & Th. & La. & ULP \\
Sandy Bridge & $P(x)$ & Fact. & Fact. & Horner & Fact. & Estrin & Horner \\
Sandy Bridge & $e^{x}$ & Fact. & Fact. & Horner & Fact. & Fact. & Horner \\
Broadell & $P(x)$ & Fact. & Fact. & Horner & Fact. & Fact. & Horner \\
Broadwell & $e^{x}$ & Fact. & Fact. & Horner & Fact. & Fact. & Horner \\
\hline
\end{tabular}

\subsection{Validation}

Given the complexity of modern microprocessors, building an efficient polynomial evaluation method is not a trivial task, neither from a theoretical point of view nor from a practical one, since vendor performance simulators are not released on the market. Nevertheless, in some special cases it is possible to explain experimental measurements with simple theoretical models. This may also serve as a validation that our measurement method produces reasonable results. For instance, application of the classical Horner's method produces a dependency chain between all the operations (as shown by the ASM DAG, Figure 2). In terms of latency, a dependency chain such as this means that the latency of each step in the critical path should be added to obtain the latency of the full method. Horner's method requires 10 successive mul/add or FMA operations, therefore the theoretical total latency of the polynomial is: $10[\text { mul+add }]_{\text {latency }}$ or $10[\mathrm{FMA}]_{\text {latency }}$ if the machine natively supports the FMA operations. Given that the latency of FMA is 5 [cycle] on Broadwell and the latency of mul+add is 8 [cycle] on Sandy Bridge [Fog 2018], we expect a latency of 50 [cycle] on Broadwell and 80 [cycle] on Sandy Bridge. The measurement gives 50.03 [cycle] on the Broadwell architecture and 80.01 [cycle] on the Sandy Bridge architecture, which corresponds to the rough model proposed previously.

Last but not least, simple factorizations evaluated using the classical Horner method can be also modeled. Considering $P_{h^{1}}^{6} P_{h^{1}}^{4}$, and given that the operations mul, add, and FMA are fully pipelined, we can assume that the computation of the polynomial of lower degree will be fully hidden by the polynomial of larger degree. In such situation, the total latency of the method is $6[\mathrm{mul}+\mathrm{add}]_{\text {latency }}+[\mathrm{mul}]_{\text {latency }}$ or $6[\mathrm{FMA}]_{\text {latency }}+[\mathrm{mul}]_{\text {latency }}$. The last multiplication corresponds to the final product between the two polynomials: it is a blocking operation. The measurement gives 36.00 [cycle] on the Broadwell architecture and 53.04 [cycle] on the Sandy Bridge, and once more the measurements correspond to our intuition. This first analysis already tells us two things: that the factorization is efficient and our performance measurement methods are correct.

\section{RESULTS}

Table 5 presents a snapshot of the evaluation methods and delivers the key results of the methods developed in this article for the evaluation of polynomials and the exponential function. From the summary in Table 5, we conclude that the factorization method yields excellent results in both Latency and Throughput (except for Latency on the Sandy Bridge Platform). However for accuracy as measured by the ULP, the Horner method is the best one. In the next sections, we explain the results in detail and present the corresponding analysis. Due to the amount of data, in tables, we only show top five results per criteria, while in figures, we point out the datapoints corresponding to state-of-the-art methods and the best factorization. 

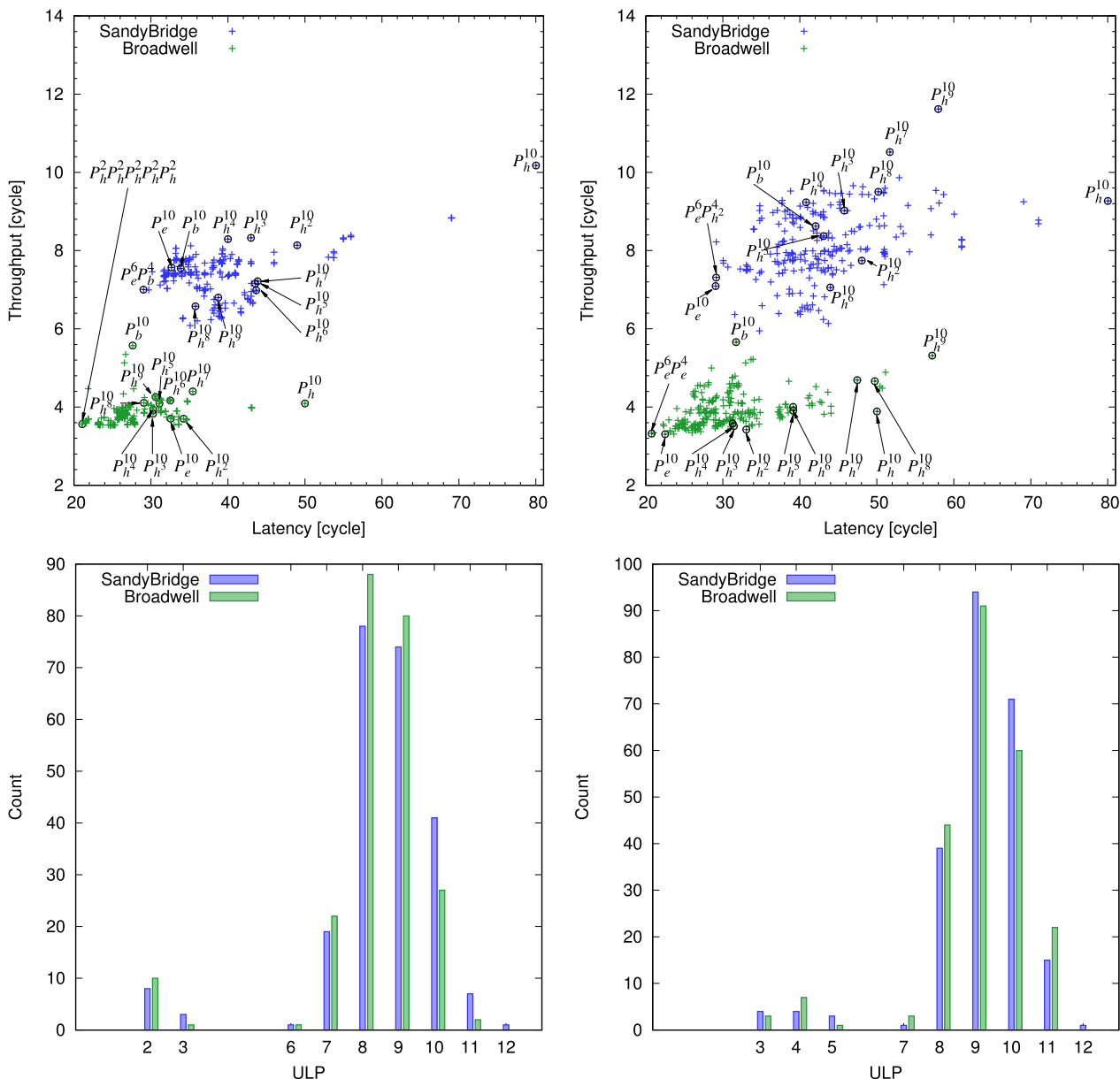

Fig. 3. Latency/Inverse Throughput of the polynomial evaluations for all architectures: scalar version (left) and vectorial version (right). In the vectorial version, the inverse throughput numbers are reported per vector instruction, thus the effective throughput per scalar operation must be obtained by dividing by the number of double precision values that fit in a vector register (e.g., 4 in the case of AVX). Histogram of the precision of all polynomial evaluation for scalar version (left) and vector version (right). The bar of the histogram is the total number of polynomial evaluations for a given ULP.

\subsection{Polynomials, Latency Analysis}

The results of the latency/throughput benchmarks for the polynomial evaluation on all platforms are presented ${ }^{3}$ in Figure 3. The figure expresses in detail all the previous methods present in Section 1 , and the best factorization method. The results are not distributed uniformly. For the scalar version the median latency values were 26.6 [cycle] (Broadwell) and 37.0 [cycle] (Sandy Bridge). For the vectorial version, the distribution is more spread out.

In detail, some clusters of points appear, as shown also in Table 6. This clustering can be ascribed to the -fast-math option, which reorganizes the computation of the power of $n$, thought

\footnotetext{
${ }^{3}$ Detailed results can be observed in the supplementary interactive figure. The interactive figure allows to select and observe every single data point.
} 
Table 6. Best Methods for the Polynomial Evaluations on Sandy Bridge and Broadwell Platforms

\begin{tabular}{|c|c|c|c|c|c|c|c|}
\hline \multicolumn{8}{|c|}{ Sandy Bridge } \\
\hline \multicolumn{5}{|c|}{ Scalar } & \multicolumn{3}{|c|}{ Vector } \\
\hline \multicolumn{5}{|c|}{ Throughput Criteria } & \multicolumn{3}{|c|}{ Throughput Criteria } \\
\hline Algorithm & Th. & La. & ULP & Algorithm & Th. & La. & ULP \\
\hline$P_{h^{4}}^{6} P_{e}^{2} P_{h^{1}}^{2}$ & 6.08 & 35.10 & 8 & $P_{b}^{2} P_{h^{2}}^{4} P_{h^{2}}^{4}$ & 5.95 & 34.77 & 9 \\
\hline$P_{h^{4}}^{6} P_{h^{1}}^{2} P_{h^{1}}^{2}$ & 6.20 & 36.03 & 8 & $P_{b}^{2} P_{h^{1}}^{4} P_{h^{1}}^{4}$ & 6.13 & 43.70 & 9 \\
\hline$P_{h^{4}}^{6} P_{h^{1}}^{4}$ & 6.23 & 37.82 & 8 & $P_{b}^{2} P_{h^{1}}^{4} P_{h^{2}}^{4}$ & 6.24 & 43.06 & 10 \\
\hline$P_{h^{4}}^{6} P_{e}^{4}$ & 6.26 & 34.12 & 8 & $P_{b}^{2} P_{e}^{4} P_{h^{2}}^{4}$ & 6.37 & 31.56 & 11 \\
\hline$P_{h^{3}}^{6} P_{e}^{2} P_{e}^{2}$ & 6.27 & 39.17 & 8 & $P_{b}^{2} P_{e}^{2} P_{e}^{2} P_{e}^{2} P_{h^{1}}^{2}$ & 6.37 & 40.05 & 9 \\
\hline \multicolumn{5}{|c|}{ Latency Criteria } & \multicolumn{3}{|c|}{ Latency Criteria } \\
\hline$P_{e}^{6} P_{b}^{4}$ & 7.00 & 29.03 & 9 & $P_{e}^{10}$ & 7.09 & 29.07 & 4 \\
\hline$P_{e}^{6} P_{e}^{4}$ & 6.99 & 29.72 & 8 & $P_{e}^{6} P_{e}^{4}$ & 8.22 & 29.15 & 10 \\
\hline$P_{h^{1}}^{2} P_{b}^{4} P_{e}^{4}$ & 7.46 & 30.16 & 9 & $P_{e}^{6} P_{h^{2}}^{4}$ & 7.32 & 29.15 & 9 \\
\hline$P_{h^{1}}^{2} P_{e}^{4} P_{h^{2}}^{4}$ & 7.12 & 31.20 & 9 & $P_{\boldsymbol{e}}^{2} P_{\boldsymbol{e}}^{4} P_{h^{2}}^{4}$ & 7.74 & 30.05 & 9 \\
\hline$P_{e}^{2} P_{b}^{4} P_{e}^{4}$ & 7.47 & 31.67 & 9 & $P_{h^{1}}^{2} P_{e}^{4} P_{h^{2}}^{4}$ & 7.68 & 30.07 & 10 \\
\hline \multicolumn{8}{|c|}{ Classical schemes } \\
\hline$P_{e}^{10}$ & 7.56 & 32.67 & 2 & $P_{e}^{10}$ & 7.09 & 29.07 & 4 \\
\hline$P_{h^{1}}^{10}$ & 10.17 & 80.00 & 2 & $P_{h^{1}}^{10}$ & 9.27 & 80.01 & 3 \\
\hline$P_{h^{2}}^{10}$ & 8.13 & 49.00 & 2 & $P_{h^{2}}^{10}$ & 7.74 & 48.04 & 3 \\
\hline$P_{h^{3}}^{10}$ & 8.32 & 42.99 & 2 & $P_{h^{3}}^{10}$ & 9.02 & 45.77 & 3 \\
\hline$P_{h^{4}}^{10}$ & 8.29 & 39.99 & 2 & $P_{h^{4}}^{10}$ & 9.23 & 40.81 & 4 \\
\hline \multicolumn{8}{|c|}{ Broadwell } \\
\hline \multicolumn{5}{|c|}{ scalar } & \multicolumn{3}{|c|}{ vector } \\
\hline & \multicolumn{4}{|c|}{ Throughput Criteria } & \multicolumn{3}{|c|}{ Throughput Criteria } \\
\hline Algorithm & Th. & La. & ULP & Algorithm & Th. & La. & ULP \\
\hline$P_{h^{1}}^{2} P_{d}^{4} P_{h^{1}}^{4}$ & 3.54 & 24.84 & 8 & $P_{e}^{6} P_{h^{1}}^{4}$ & 3.31 & 23.62 & 8 \\
\hline$P_{b}^{2} P_{e}^{4} P_{h^{1}}^{4}$ & 3.54 & 24.71 & 10 & $P_{e}^{10}$ & 3.31 & 22.50 & 3 \\
\hline$P_{e}^{6} P_{h^{1}}^{4}$ & 3.54 & 24.45 & 7 & $P_{e}^{6} P_{e}^{4}$ & 3.33 & 20.75 & 9 \\
\hline$P_{e}^{6} P_{e}^{2} P_{e}^{2}$ & 3.54 & 25.30 & 8 & $P_{e}^{6} P_{h^{2}}^{4}$ & 3.33 & 21.05 & 9 \\
\hline$P_{b}^{2} P_{b}^{4} P_{h^{1}}^{4}$ & 3.54 & 24.73 & 9 & $P_{h^{2}}^{6} P_{h^{2}}^{4}$ & 3.34 & 26.01 & 8 \\
\hline \multicolumn{5}{|c|}{ Latency Criteria } & \multicolumn{3}{|c|}{ Latency Criteria } \\
\hline$P_{h^{1}}^{2} P_{h^{1}}^{2} P_{h^{1}}^{2} P_{h^{1}}^{2} P_{h^{1}}^{2}$ & 3.56 & 21.04 & 8 & $P_{e}^{6} P_{e}^{4}$ & 3.33 & 20.75 & 9 \\
\hline$P_{e}^{2} P_{h^{1}}^{2} P_{h^{1}}^{2} P_{h^{1}}^{2} P_{h^{1}}^{2}$ & 3.56 & 21.08 & 8 & $P_{e}^{6} P_{h^{2}}^{4}$ & 3.33 & 21.05 & 9 \\
\hline$P_{b}^{2} P_{h^{1}}^{2} P_{h^{1}}^{2} P_{h^{1}}^{2} P_{h^{1}}^{2}$ & 3.56 & 21.08 & 9 & $P_{e}^{2} P_{e}^{4} P_{h^{2}}^{4}$ & 3.55 & 22.25 & 11 \\
\hline$P_{b}^{2} P_{b}^{2} P_{e}^{2} P_{e}^{2} P_{h^{1}}^{2}$ & 3.64 & 21.22 & 9 & $P_{h^{1}}^{2} P_{e}^{4} P_{h^{2}}^{4}$ & 3.51 & 22.33 & 10 \\
\hline$P_{b}^{2} P_{b}^{2} P_{b}^{2} P_{e}^{2} P_{h^{1}}^{2}$ & 3.64 & 21.23 & 8 & $P_{e}^{10}$ & 3.31 & 22.50 & 3 \\
\hline \multicolumn{8}{|c|}{ Classical schemes } \\
\hline$P_{e}^{10}$ & 3.70 & 32.57 & 3 & $P_{e}^{10}$ & 3.31 & 22.50 & 3 \\
\hline$P_{h^{1}}^{10}$ & 4.09 & 50.00 & 3 & $P_{h^{1}}^{10}$ & 3.34 & 50.01 & 2 \\
\hline$P_{h^{2}}^{10}$ & 3.70 & 34.23 & 3 & $P_{h^{2}}^{10}$ & 3.42 & 33.05 & 3 \\
\hline$P_{h^{3}}^{10}$ & 3.82 & 30.26 & 2 & $P_{h^{3}}^{10}$ & 3.51 & 31.44 & 3 \\
\hline$P_{h^{4}}^{10}$ & 3.93 & 30.31 & 4 & $P_{h^{4}}^{10}$ & 3.58 & 31.27 & 3 \\
\hline
\end{tabular}

Out of all 231 possible combinations, only the top five are shown. The criteria indicates the sort of the tuple (throughput, latency, ulp) on a specific part. Classical schemes complete this table. For simplicity, we only report the most common evaluation patterns.

ACM Transactions on Mathematical Software, Vol. 46, No. 3, Article 28. Publication date: September 2020. 
to be impossible for the vector versions, as all the versions are slightly different (we confirmed this by a quick look at the disassembled code). Considering the out-of-order execution and the renaming of the register, it is clear that a few versions give the same results. The phenomenon is especially visible for the schemes that contain polynomials of type $P_{b}$ that are evaluated with the "brute force" method. It appears that the compiler has the ability to aggressively optimize the pattern $x^{n}$, but not the composition of function $f(f(x))$ (used in Estrin and Horner method). Consequently, for schemes that contain monomial evaluation using the "brute force method," the benchmark may reflect the tree optimizer of the compiler more than the desired algorithm. In contrast, these clusters are less visible in the vectorial version, because SIMD intrinsics programming prevents any optimization of the -fast-math, i.e., every algorithm has a unique execution path.

Considering the Horner method as the historical reference point, the spectra of results are large. For the Latency measurement, as expected for all platforms the longest latency is observed with the classical Horner method due to the chain dependency. The other Horner $k$ th order method shows a nice improvement until the 4th order (range 30-35 [cycle] on Broadwell and 40-50 [cycle] on Sandy Bridge), beyond which the performance decrease due to the influence of computing the power of the main monomial factor $x^{n}$, which becomes the bottleneck.

The brute force method provides nice results, thanks the evaluation of the power and the storage of the intermediate power associated to the superscalar structure of the processor. The latencies are equivalent to the best Horner method (4th order).

The Estrin method is interesting, because it can be considered like the state-of-the-art. Results are excellent, but not the best ones. We remark that, for the Broadwell architecture, Estrin's method is slower $(10$ [cycles]) than Sandy Bridge, which is not satisfying. It seems that the -fast-math option could be problematic for this pattern on this platform. For Sandy Bridge Estrin it is the best method, but the difference with the factorization is lower than the throughput of an addition/multiplication operation.

Finally, the factorization pattern provides the best results (except for Sandy Bridge, where it is equivalent to Estrin's method). These results are the most complex to analyze, because they are dispatched all over the figures. Two patterns seem important: (1) The product of two single polynomials associated to Estrin, e.g., $P_{e}^{6} P_{e}^{4}$. (2) A full product of quadratics. On this last point, especially on Broadwell, FMA and MUL are computed on the same FPU circuit (FMA) and they are fully pipelined. Consequently, these two mnemonics can be executed with a minimum inverse throughput of 0.5 [cycle] if they are independent. With two FPUs, this means that the $\mu$ ops scheduler should dispatch the instruction over the two FPUs by limiting the dependency to the minimum, i.e., when two quadratics have been computed on each FPU, their product starts immediately on an FPU and a new quadratic starts on the other FPU. The factorization and the dependency chain become difficult to predict.

In the vectorial version on Sandy Bridge, the factorization method is second, but the difference with the factorization is lower than the throughput of an addition/multiplication, so the methods can be considered equivalent. On Broadwell, the factorization is up to 1.75 cycles faster than the Estrin method (speedup of 8\%). This speedup represents more than three times the throughput of the FMA or multiplication, and it should be considered as the direct effect of the parallelism exposed by the factorization. In the scalar version, factorization outperforms other methods. The method is 3 cycles faster on Sandy Bridge and up to 10 cycles faster on Broadwell (but this can be considered as an unfair comparison, as we demonstrated before). In general, the complementarity of the superscalar architecture and the code generated by the compiler using -fast-math can sustain very good performance. 
Table 7. Benchmark of the $2^{k}$ and the Boundary Conditions for the Evaluation of $e^{x}$

\begin{tabular}{lcccccccc}
\hline Architecture & \multicolumn{3}{c}{$2^{k}$} & \multicolumn{4}{c}{ boundary } \\
& \multicolumn{2}{c}{ scalar } & \multicolumn{2}{c}{ vector } & \multicolumn{3}{c}{ scalar } & \multicolumn{2}{c}{ vector } \\
& Thr. & Lat. & Thr. & Lat. & Thr. & Lat. & Thr. & Lat. \\
Sandy Bridge & 4.71 & 15.00 & 3.49 & 18.99 & 4.71 & 9.95 & 4.04 & 8.01 \\
Broadwell & 3.63 & 16.00 & 2.70 & 18.00 & 4.94 & 10.65 & 2.64 & 8.01 \\
\hline
\end{tabular}

\subsection{Polynomials, Inverse Throughput Analysis}

In the case of vector operations, we report the inverse throughput per vector operation. To obtain the throughput per scalar operation, one must divide our measurements by the number of doubleprecision floating point variables that fit in a vector register. For example, the vectorial inverse throughput of 3.31 for $P_{e}^{6} P_{h^{1}}^{4}$ reported in Table 6 should be divided by 4 to obtain the throughput per scalar operation, because 4 double-precision floating point variables fit in a single AVX vector register.

By looking at Table 6, it is clear that the factorization and all parallel patterns slightly decrease the throughput. An explanation for this can be that the factorization pattern improves the parallelism and consequently a better filling of the pipeline, especially on the Broadwell architecture, as described in the previous section.

\subsection{Polynomials, Precision Analysis}

Results from ULP error analysis are presented in Figure 3. Polynomial schemes can be categorized in two groups according to the Unit of Least Precision (ULP): the first group has a small ULP (2 to 5 ) while the second a larger ULP (larger than 6). This behavior is easily explained. The first group concerns all evaluation schemes of type $P_{m}^{10}$, with no factorization applied; in contrast, the 2nd group contains schemes in which factorization was applied. Since the computation of the coefficients in the factorization process requires solving quadratic equations, it introduces numerical errors during the manipulation of the determinant due to rounding error. In this work, we used the MATLAB software in this step of the process. In Kahan [2002] the phenomenon of error propagation on simple quadratic equations is studied and some techniques to reduce round-off errors are provided, consequently, it should be possible to improve the ULP of all factorization patterns.

More surprisingly, the vector presents a shift of 1 ULP compared to the scalar version. However, we do not have a clear explanation for this shift. In the scalar version the compiler has more freedom to reorganize operations than the vector version where the SIMD intrinsics associated with the algorithm fix the operation's execution. In the scalar version, the compiler reorganizes the operations following policy setting, which we believe is able to maximize the performance while minimizing the error.

\subsection{Exponentials}

Evaluating the exponential requires the addition of the computation of $2^{k}$ and the boundary conditions that have been described in Sections 2.3 and 2.4. The inverse throughput/latency benchmarks are reported in Table 7. The results are similar regardless of the platform or backend (vectorial/scalar), with a small improvement on the Broadwell version, especially for the vector version, because it supports AVX2, which allows more vectorial SIMD integer instructions. The computation of the boundary conditions and $2^{k}$ are quite expensive in terms of latency cycles. This is easily explained by the fact that both operations introduce a chain of dependencies that does not allow parallelism, despite the large number of ALUs inside the processors. 

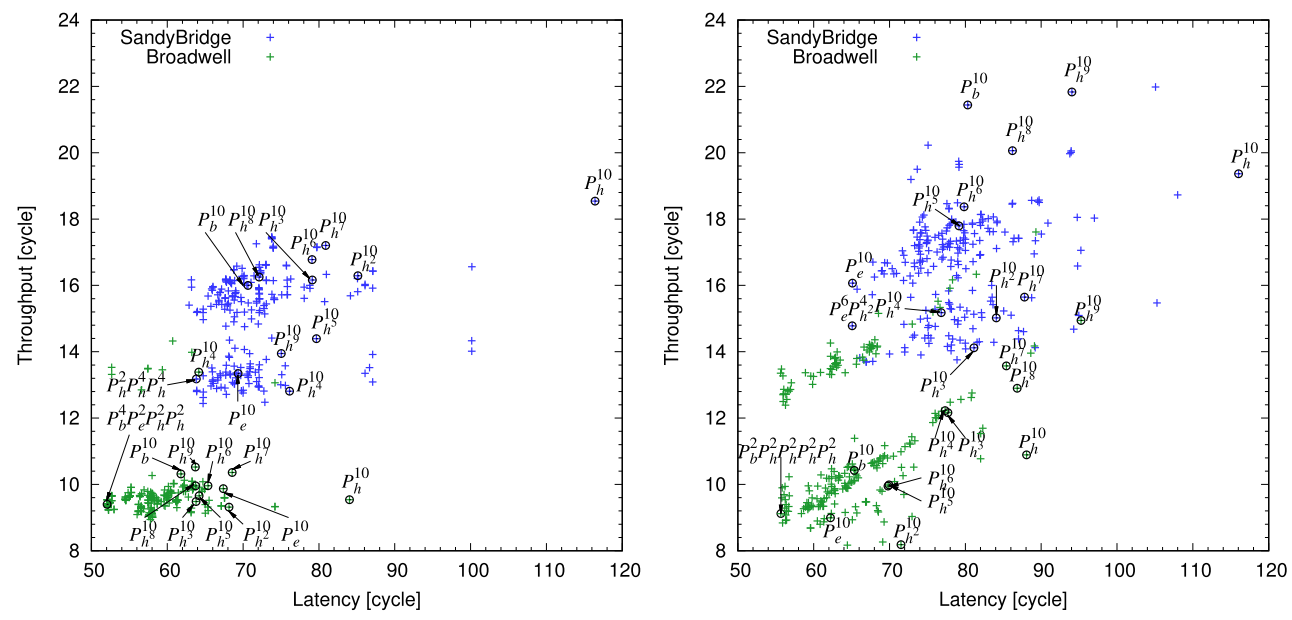

Fig. 4. Latency/Throughput of $e^{x}$ for all architectures: scalar version (left) and vectorial version (right).

Globally, evaluating the full $e^{x}$ function resulted in the best measurements of the inverse throughput being multiplied by a factor of roughly 3 and the latency by a factor of roughly 2 , compared to the bare evaluation of the polynomials. Figure 4 and Table 8 confirm the results, already obtained by polynomial evaluation, that the factorization scheme provides the best performance for both throughput and latency. However, interestingly, the results do not reproduce perfectly the pattern of the polynomial evaluation. The addition of the boundary conditions and $2^{k}$ showed better performance for exponentials based on the products of quadratics instead of the Estrin method or products of higher degree polynomials. It is difficult to provide a clear reason, but we believe that the higher degree of parallelism exposed by the factorization into products of quadratics may allow the out-of-order engine a much larger space for optimization, thus leading to better performance. For the Sandy Bridge platform the latency of the Estrin method is very close to the factorization. In detail, the factorizations provide good results due to better filling of the pipelines. Consequently, the ports 0,1 (floating point) are saturated, which slows down the integer and bitwise operations for the boundary conditions and the evaluation of $2^{k}$, which are also evaluated on similar ports ( 0,1 and 5$)$, and subsequently throughput increases drastically for this scheme. The larger number of port and FPU/APUs limit this effect on Broadwell compared to Sandy Bridge. Moreover, this effect is mainly presented on the vectorial version, because the SIMD code is generated in such a way that the intrinsics prevent any optimization of the compiler due to the -fast-math option compared to the scalar version. However, it is difficult to formulate a clear explanation of the complicated performance relationship between the computation of the polynomial, $2^{k}$, and the boundaries, due to the out-of-order execution. In such context, as every method's performance is unpredictable and independent of other methods, the benchmark is the only way to determine the best evaluation pattern.

For the errors, there is no difference with the polynomials errors because the computation of $2^{k}$ and the boundary limit are guaranteed to be error-free.

The final comparison concerns the Intel Mathematical Function (IMF - scalar version) and Intel Short Vector Mathematical Library (SVML - vectorial version), both libraries are delivered with the Intel Compiler (15.0.0). We benchmarked the out-of-the-box implementation of the exponential function from these libraries. The benchmarks [throughtput/latency/ulp] provide the following results for the scalar [20.61/52.69/2] and vectorial version [6.38/39.20/2] on Broadwell and scalar 
Table 8. Best Latency/throughput [Cycle] for the Evaluation of the Full $e^{x}$ Function on the Sandy Bridge Platform, Including Boundary Conditions and Computation of $2^{k}$ as Described in Sections 2.3 and 2.4

\begin{tabular}{|c|c|c|c|c|c|c|c|}
\hline \multicolumn{8}{|c|}{ Sandy Bridge } \\
\hline \multicolumn{5}{|c|}{ scalar } & \multicolumn{3}{|c|}{ vector } \\
\hline \multicolumn{5}{|c|}{ Throughput Criteria } & \multicolumn{3}{|c|}{ Throughput Criteria } \\
\hline Algorithm & Th. & La. & ULP & Algorithm & Th. & La. & ULP \\
\hline$P_{e}^{2} P_{b}^{4} P_{h^{2}}^{4}$ & 12.44 & 64.73 & 9 & $P_{e}^{6} P_{b}^{4}$ & 13.69 & 66.92 & 9 \\
\hline$P_{h^{2}}^{6} P_{e}^{2} P_{h^{1}}^{2}$ & 12.48 & 72.88 & 7 & $P_{b}^{2} P_{e}^{4} P_{e}^{4}$ & 13.69 & 66.92 & 11 \\
\hline$P_{b}^{2} P_{e}^{4} P_{h^{2}}^{4}$ & 12.62 & 64.75 & 9 & $P_{e}^{2} P_{e}^{4} P_{h^{2}}^{4}$ & 13.71 & 67.79 & 9 \\
\hline$P_{b}^{2} P_{h^{2}}^{4} P_{h^{2}}^{4}$ & 12.72 & 67.79 & 9 & $P_{e}^{2} P_{h^{1}}^{4} P_{h^{1}}^{4}$ & 13.76 & 78.90 & 9 \\
\hline$P_{h^{3}}^{6} P_{h^{1}}^{2} P_{h^{1}}^{2}$ & 12.76 & 70.01 & 9 & $P_{h^{1}}^{2} P_{e}^{4} P_{h^{2}}^{4}$ & 13.82 & 66.08 & 10 \\
\hline \multicolumn{5}{|c|}{ Latency Criteria } & \multicolumn{3}{|c|}{ Latency Criteria } \\
\hline$P_{h^{1}}^{2} P_{b}^{4} P_{e}^{4}$ & 15.40 & 62.88 & 9 & $P_{e}^{6} P_{h^{2}}^{4}$ & 14.78 & 65.08 & 9 \\
\hline$P_{e}^{2} P_{e}^{4} P_{e}^{4}$ & 16.17 & 63.19 & 10 & $P_{e}^{10}$ & 16.07 & 65.12 & 3 \\
\hline$P_{b}^{2} P_{e}^{4} P_{e}^{4}$ & 15.95 & 63.21 & 10 & $P_{e}^{6} P_{e}^{4}$ & 15.88 & 65.72 & 9 \\
\hline$P_{h^{1}}^{2} P_{e}^{4} P_{e}^{4}$ & 13.18 & 63.84 & 9 & $P_{h^{1}}^{2} P_{e}^{4} P_{h^{2}}^{4}$ & 13.82 & 66.08 & 10 \\
\hline$P_{e}^{2} P_{b}^{4} P_{e}^{4}$ & 12.80 & 63.88 & 9 & $P_{b}^{2} P_{e}^{4} P_{e}^{4}$ & 13.69 & 66.92 & 11 \\
\hline \multicolumn{8}{|c|}{ Classical schemes } \\
\hline$P_{e}^{10}$ & 13.34 & 69.35 & 2 & $P_{e}^{10}$ & 16.07 & 65.12 & 3 \\
\hline$P_{h^{1}}^{10}$ & 18.54 & 116.41 & 2 & $P_{h^{1}}^{10}$ & 19.37 & 116.02 & 3 \\
\hline$P_{h^{2}}^{10}$ & 16.29 & 85.13 & 2 & $P_{h^{2}}^{10}$ & 15.02 & 84.07 & 3 \\
\hline$P_{h^{3}}^{10}$ & 16.16 & 79.13 & 2 & $P_{h^{3}}^{10}$ & 14.12 & 81.12 & 3 \\
\hline$P_{h^{4}}^{10}$ & 12.81 & 76.13 & 2 & $P_{h^{4}}^{10}$ & 15.18 & 76.00 & 4 \\
\hline
\end{tabular}

Broadwell

\begin{tabular}{|c|c|c|c|c|c|c|c|}
\hline Algorithm & Th. & La. & ULP & Algorithm & Th. & La. & ULP \\
\hline$P_{e}^{6} P_{h^{1}}^{4}$ & 8.94 & 57.77 & 8 & $P_{h^{2}}^{6} P_{b}^{4}$ & 8.17 & 64.44 & 9 \\
\hline$P_{h^{1}}^{2} P_{h^{1}}^{4} P_{h^{1}}^{4}$ & 9.01 & 58.05 & 9 & $P_{h^{2}}^{10}$ & 8.18 & 71.52 & 3 \\
\hline$P_{b}^{2} P_{h^{1}}^{4} P_{h^{1}}^{4}$ & 9.01 & 58.05 & 9 & $P_{h^{2}}^{8} P_{b}^{2}$ & 8.26 & 69.10 & 8 \\
\hline$P_{e}^{2} P_{h^{1}}^{4} P_{h^{1}}^{4}$ & 9.01 & 58.05 & 8 & $P_{b}^{2} P_{b}^{4} P_{h^{2}}^{4}$ & 8.68 & 59.60 & 10 \\
\hline$P_{h^{1}}^{2} P_{e}^{4} P_{h^{1}}^{4}$ & 9.03 & 57.55 & 8 & $P_{e}^{6} P_{h^{2}}^{4}$ & 8.68 & 59.57 & 9 \\
\hline \multicolumn{5}{|c|}{ Latency Criteria } & \multicolumn{3}{|c|}{ Latency Criteria } \\
\hline$P_{b}^{4} P_{e}^{2} P_{h^{1}}^{2} P_{h^{1}}^{2}$ & 9.40 & 52.07 & 10 & $P_{b}^{2} P_{b}^{2} P_{b}^{2} P_{b}^{2} P_{e}^{2}$ & 13.35 & 55.65 & 10 \\
\hline$P_{e}^{4} P_{b}^{2} P_{h^{1}}^{2} P_{h^{1}}^{2}$ & 9.42 & 52.07 & 9 & $P_{b}^{2} P_{h^{1}}^{2} P_{h^{1}}^{2} P_{h^{1}}^{2} P_{h^{1}}^{2}$ & 9.12 & 55.67 & 9 \\
\hline$P_{b}^{4} P_{b}^{2} P_{h^{1}}^{2} P_{h^{1}}^{2}$ & 9.42 & 52.07 & 9 & $P_{b}^{2} P_{b}^{2} P_{b}^{2} P_{b}^{2} P_{h^{1}}^{2}$ & 13.48 & 55.76 & 9 \\
\hline$P_{e}^{4} P_{e}^{2} P_{h^{1}}^{2} P_{h^{1}}^{2}$ & 9.42 & 52.08 & 9 & $P_{b}^{2} P_{e}^{2} P_{h^{1}}^{2} P_{h^{1}}^{2} P_{h^{1}}^{2}$ & 9.60 & 55.80 & 10 \\
\hline$P_{b}^{2} P_{b}^{2} P_{e}^{2} P_{h^{1}}^{2} P_{h^{1}}^{2}$ & 9.53 & 52.09 & 8 & $P_{b}^{2} P_{b}^{2} P_{h^{1}}^{2} P_{h^{1}}^{2} P_{h^{1}}^{2}$ & 9.90 & 55.86 & 9 \\
\hline \multicolumn{8}{|c|}{ Classical schemes } \\
\hline$P_{e}^{10}$ & 9.87 & 67.39 & 2 & $P_{e}^{10}$ & 8.99 & 62.22 & 3 \\
\hline$P_{h^{1}}^{10}$ & 9.54 & 84.03 & 2 & $P_{h^{1}}^{10}$ & 10.89 & 88.07 & 3 \\
\hline$P_{h^{2}}^{10}$ & 9.32 & 68.14 & 2 & $P_{h^{2}}^{10}$ & 8.18 & 71.52 & 3 \\
\hline$P_{h^{3}}^{10}$ & 9.48 & 63.79 & 2 & $P_{h^{3}}^{10}$ & 9.98 & 69.92 & 3 \\
\hline$P_{h^{4}}^{10}$ & 13.39 & 64.16 & 2 & $P_{h^{4}}^{10}$ & 9.95 & 69.79 & 4 \\
\hline
\end{tabular}

Out of all 231 possible combinations, only the top five are shown. The criteria indicate the sort of the tuple (throughput, latency, ulp) on a specific part. Classical schemes complete this table. For simplicity, we only report the most common evaluation patterns. 
[12.11/67.39/2], vectorial [12.25/55.37/2] on Sandy Bridge. Comparing these numbers to our results in Table 8 shows that for some evaluation patterns, our implementation can be equivalent or superior, especially for the scalar version on Sandy Bridge platform. However, the vectorial on Broadwell is not reachable. Since the Sandy Bridge platform is quite old (the first products were released in 2011), Intel may have not updated their library for this target. Our results have a larger error, which we know comes from the factorization pattern. For the difference of performance between latency and throughput, we have no information about Intel implementation, since Intel does not communicate about this. Unfortunately, we have no valid explanation for the poor performance on the IMF library, since a good vectorial version such as the SVML should automatically give good results for the scalar version, as the registers are the same (XMM is nested in YMM).

\section{CONCLUSIONS}

In this article, we evaluated the most famous polynomial pattern on 10 degree polynomials; moreover, we explored the factorization scheme with systematic combinations of all patterns. We did a systematic study of the three key parameters of [throughput/latency/ulp]. Processors have become so complex that an approach based on the complexity of the algorithm is not enough. For methods whose complexity becomes difficult to estimate, such as the factorization pattern, benchmarks are mandatory to determine the best-performing solution. Thus, we showed that factorization is an interesting pattern on superscalar architecture and it can provide better results for both throughput and latency. Unfortunately, although the -fast-math is an interesting option for code not generated by a DSL, it can have an undesired effect on efficient patterns (Estrin).

We demonstrated a dependency between throughput and latency of polynomial evaluation techniques on modern superscalar processors. Our results are similar to the vendor libraries, although we do not have a clear idea of the vendors' algorithm. However, factorization definitively appears to be a good pattern on superscalar processor and could be applied to other fields of computer science.

To speed up computations, diminishing the degree of the polynomial of the approximation is an option. The precision will be impacted but some applications may not need high precision. Future research could explore the influence of the polynomial degree on performance and precision and should focus on the comparison with preconditioned methods on the needed polynomial for $e^{x}$.

\section{ACKNOWLEDGMENTS}

The authors would like to gratefully Sam Yates for the latency measurement library and all the members of the Blue Brain Project HPC team for the many discussions and feedback they provided. In addition, we would like to thank the Swiss National Supercomputing Center (CSCS) and their collaborators (Hussein Nasser El- Harake) for providing access and support to a Broadwell system, and last but not least, Martina Scolamiero (BBP) and Vivien Lecomte (from Laboratoire de Probabilités et Modèles Aléatoires, LPMA UMR 7599) for their mathematical support.

\section{REFERENCES}

Muhammad Abbas and Oscar Gustafsson. 2011. Computational and implementation complexity of polynomial evaluation schemes. In Proceedings of the NORCHIP Conference. IEEE, 1-6.

George A. Baker and Peter Graves-Morris. 1996. Padé Approximants (2nd ed.). Cambridge University Press. DOI : https:// doi.org/10.1017/CBO9780511530074

Prasanna Balaprakash, Jack Dongarra, Todd Gamblin, Mary Hall, Jeffrey K. Hollingsworth, Boyana Norris, and Richard Vuduc. 2018. Autotuning in high-performance computing applications. Proc. IEEE 99 (2018), 1-16.

M. Boersma, M. Kroner, C. Layer, P. Leber, S. M. Muller, and K. Schelm. 2011. The POWER7 binary floating-point unit. In Proceedings of the 20th IEEE Symposium on Computer Arithmetic (ARITH'11). 87-91.

T. Agerwala and J. Cocke. 1987. High Performance Reduced Instruction Set Processors. IBM Watson Research Center. 
S. Chevillard, M. Joldeş, and C. Lauter. 2010. Sollya: An environment for the development of numerical codes. In Mathematical Software - ICMS 2010 (Lecture Notes in Computer Science), K. Fukuda, J. van der Hoeven, M. Joswig, and N. Takayama (Eds.), Vol. 6327. Springer,Germany, 28-31.

Hugues de Lassus Saint-Genies, David Defour, and Guillaume Revy. 2017. Exact lookup tables for the evaluation of trigonometric and hyperbolic functions. IEEE Trans. Comput. 66, 12 (2017), 2058-2071.

W. S. Dorn. 1962. Generalizations of Horner's rule for polynomial evaluation. IBM J. Res. Dev. 6, 2 (Apr. 1962), $239-245$.

Marat Dukhan and Richard W. Vuduc. 2013. Methods for high-throughput computation of elementary functions. In Proceedings of the 10th International Conference on Parallel Processing and Applied Mathematics (PPAM'13), Revised Selected Papers, Part I. 86-95.

Milos D. Ercegovac. 1977. A general hardware-oriented method for evaluation of functions and computations in a digital computer. IEEE Trans. Comput. 7 (1977), 667-680.

Gerald Estrin. 1960. Organization of computer systems-The fixed plus variable structure computer. In Proceedings of the International Workshop on Managing Requirements Knowledge. 33.

Timothée Ewart, Fabien Delalondre, and Felix Schürmann. 2014. Cyme: A library maximizing SIMD computation on userdefined containers. In Supercomputing, Julian Martin Kunkel, Thomas Ludwig, and Hans Werner Meuer (Eds.). Lecture Notes in Computer Science, Vol. 8488. Springer International Publishing, 440-449.

Timothée Ewart, Stuart Yates, Francesco Cremonesi, Pramod Kumbhar, Felix Schürmann, and Fabien Delalondre. 2015. Performance evaluation of the IBM POWER8 architecture to support computational neuroscientific application using morphologically detailed neurons. In Proceedings of the 6th International Workshop on Performance Modeling, Benchmarking, and Simulation of High Performance Computing Systems (PMBS'15). ACM, New York, NY.

Richard J. Fateman. 2002. Code generation: Evaluating polynomials. University of California, Berkeley. Retrieved from http://people.eecs.berkeley.edu/ fateman/papers/polyval.pdf.

Agner Fog. 1996-2016. The microarchitecture of Intel, AMD and VIA CPUs An optimization guide for assembly programmers and compiler makers. Retrieved from http://www.agner.org/optimize/microarchitecture.pdf.

Agner Fog. 2018. Instruction tables. Retrieved from http://www.agner.org/optimize/instruction_tables.pdf.

W. Fraser. 1965. A survey of methods of computing minimax and near-minimax polynomial approximations for functions of a single independent variable. F. ACM 12, 3 (July 1965), 295-314.

Curtis F. Gerald and Patrick O. Wheatley. 2004. Applied Numerical Analysis. Pearson/Addison-Wesley.

David Goldberg. 1991. What every computer scientist should know about floating-point arithmetic. ACM Comput. Surv. 23, 1 (March 1991), 5-48.

Kazushige Goto and Robert A. van de Geijn. 2008. Anatomy of high-performance matrix multiplication. ACM Trans. Math. Softw. 34, 3 (May 2008).

HiPEAC 2015. Fast Exponential Computation on SIMD Architectures. HiPEAC.

Intel. 2009-2012. Intel Architecture Code Analyser. Retrieved from https://software.intel.com/en-us/articles/intelarchitecture-code-analyzer.

Mioara Joldes, Jean-Michel Muller, and Valentina Popescu. 2017. Tight and rigorous error bounds for basic building blocks of double-word arithmetic. ACM Trans. Math. Softw. 44, 2 (Oct. 2017). DOI : https://doi.org/10.1145/3121432

W. Kahan. 2002. On the Cost of Floating-point Computation without Extra-precise Arithmetic. Retrieved from https:// people.eecs.berkeley.edu/ wkahan/Qdrtcs.pdf.

Felix Klein. 1932. Elementary Mathematics from an Advanced Standpoint. MacMillan and Co. Limited.

Donald E. Knuth. 1962. Evaluation of polynomials by computer. Commun. ACM 5, 12 (1962), 595-599.

Donald E. Knuth. 1997. The Art of Computer Programming, Volume 2 (3rd ed.): Seminumerical Algorithms. Addison-Wesley Longman Publishing Co., Inc., Boston, MA.

Monica S. Lam. 1990. Instruction scheduling for superscalar architectures. Annu. Rev. Comput. Sci. 4 (1990), $173-201$.

C. Lauter. 2016. A new open-source SIMD vector libm fully implemented with high-level scalar C. In Proceedings of the 50th Asilomar Conference on Signals, Systems and Computers. 407-411.

Christoph Quirin Lauter. 2005. Basic Building Blocks for a Triple-double Intermediate Format. Technical Report RR-5702. INRIA. Retrieved from https://hal.inria.fr/inria-00070314.

Richard J. Lipton and Larry J. Stockmeyer. 1978. Evaluation of polynomials with super-preconditioning. 7. Comput. Syst. Sci. 16, 2 (1978), 124-139.

Sparsh Mittal. 2018. A Survey of Techniques for Dynamic Branch. Retrieved from https://arxiv.org/abs/1804.00261.

S. L. Moshier. 2000. Cephes Math Library. Retrieved from http://www.moshier.net.

Christophe Mouilleron and Guillaume Revy. 2011. Automatic generation of fast and certified code for polynomial evaluation. In Proceedings of the 20th IEEE Symposium on Computer Arithmetic (ARITH'11). IEEE, 233-242.

Jean-Michel Muller. 1997. Elementary Functions: Algorithms and Implementation. Birkhauser Boston, Inc., Secaucus, NJ.

Jean-Michel Muller. 2005. On the Definition of ulp(x). Retrieved from http:/www.ens-lyon.fr/LIP/Pub/Rapports/RR/ RR2005/RR2005-09.pdf. 
Jean-Michel Muller. 2006. Elementary Functions. Springer.

A. C. R. Newbery. 1975. Polynomial evaluation schemes. Math. Comp. 29, 132 (1975), 1046-1050.

Richard E. Overill and Stephen Wilson. 1994. Performance of parallel algorithms for the evaluation of power series. Parallel Comput. 20, 8 (1994), 1205-1213.

Angela Pohl, Biagio Cosenza, Mauricio Alvarez Mesa, Chi Ching Chi, and Ben Juurlink. 2016. An evaluation of current SIMD programming models for $\mathrm{C}++$. In Proceedings of the 3rd Workshop on Programming Models for SIMD/Vector Processing (WPMVP'16). ACM, New York, NY.

Michael O. Rabin and Shmuel Winograd. 1972. Fast evaluation of polynomials by rational preparation. Commun. Pure Appl. Math. 25, 4 (1972), 433-458.

Gavin S. Reynolds. 2010. Investigation of Different Methods of Fast Polynomial Evaluation. Master's thesis. The University of Edinburgh.

Hugues De Lassus Saint-Genies. 2018. Elementary Functions: Towards Automatically Generated, Efficient, and Vectorizable Implementations. Ph.D. Dissertation. Université de Perpignan.

Naoki Shibata. 2010. Efficient evaluation methods of elementary functions suitable for SIMD computation. Comput. Sci. Res. Dev. 25, 1 (2010), 25-32.

Lol Software. 2012. Remez exchange toolbox. Retrieved from http://lolengine.net/wiki/doc/maths/remez.

Ping-Tak Peter Tang. 1989. Table-driven implementation of the exponential function in IEEE floating-point arithmetic. ACM Trans. Math. Softw. 15, 2 (June 1989), 144-157.

P. T. P. Tang. 1991. Table-lookup algorithms for elementary functions and their error analysis. In Proceedings of the 10th IEEE Symposium on Computer Arithmetic. 232-236.

David Vandevoorde and Nicolai M. Josuttis. 2002. C++ Templates: The Complete Guide(1st ed.). Addison-Wesley Professional.

Received July 2018; revised September 2019; accepted June 2020 